\title{
La nutrizione nel paziente oncologico: a che punto siamo?
}

\author{
Ludovica Cogorno $^{1} \cdot$ Eleonora Poggiogalle ${ }^{1} \cdot$ Lorenzo M. Donini $^{1}$
}

Accettato: 8 dicembre 2021 / Pubblicato online: 21 febbraio 2022

(c) The Author(s) 2022

\section{Sommario}

La prima osservazione che la malnutrizione e la perdita di peso contribuiscano alla morte dei malati di cancro in maniera significativa e indipendentemente dagli effetti del tumore stesso risale a più di 70 anni fa. Da allora l'intervento nutrizionale, inteso sia come screening sia come trattamento medico orientato alla ricerca e alla cura della malnutrizione, ha assunto sempre più significato nella gestione della patologia tumorale. Ciò sia al suo esordio sia durante la naturale evoluzione della malattia, anche quando accompagnata dai trattamenti medici convenzionali (radioterapia, chemioterapia, chirurgia e cure palliative). La nutrizione artificiale nelle sue diverse declinazioni (supplementi nutrizionali orali, nutrizione enterale e parenterale), la farmacoterapia, il protocollo enhanced recovery after surgery (ERAS) e l'immunonutrizione sono ad oggi gli strumenti a nostra disposizione per invertire o almeno arrestare il processo prima che sopraggiunga la cachessia. Questa breve rassegna nasce con l'intento di descrivere a che punto siamo nel trattamento nutrizionale del paziente oncologico e quali sono ad oggi le evidenze di efficacia dei vari interventi nei diversi momenti di malattia.

Parole chiave Malnutrizione $\cdot$ Neoplasia $\cdot$ Intervento nutrizionale

\section{Introduzione}

Le malattie neoplastiche rappresentano la seconda causa di morte in tutto il mondo e si prevede che il numero di nuovi casi aumenti significativamente nei prossimi decenni [1].

La malnutrizione è una condizione comune nei malati oncologici ed è la conseguenza sia della presenza del tumore stesso sia delle cure mediche e chirurgiche antitumorali a cui si ricorre. Si tratta di una condizione con un impatto negativo sulla qualità della vita e sulla tossicità del trattamento. Si stima che fino al 10-20\% dei malati di cancro muoia a causa delle conseguenze della malnutrizione piuttosto che per il tumore stesso [1].

Fino all' $80 \%$ dei pazienti affetti da neoplasie maligne in stadio avanzato incorre nella cosiddetta sindrome da cachessia tumore associata (CCS), lo stato estremo della malnutrizione. Con questa ci si riferisce al complesso stato metabolico che porta all'esaurimento di energia e di riserve sia adipose sia muscolari in modo irreversibile, spesso anche a

\footnotetext{
Proposto da L.M. Donini.

L.M. Donini

lorenzomaria.donini@uniroma1.it

1 Dipartimento di Medicina Sperimentale, Sapienza Università di Roma, Roma, Italia
}

fronte di un intervento con un adeguato piano nutrizionale [2].

Il primo segno dell'evoluzione verso un quadro di malnutrizione è la perdita di peso, la cui entità ha un significato prognostico importante, specialmente se questa avviene prima di qualsiasi trattamento chirurgico, radioterapico o chemioterapico. La più alta incidenza di calo ponderale si osserva nei tumori gastrici e pancreatici (85\%) [2]. A seguire i tumori del colon, della prostata, del polmone e i linfomi più aggressivi nei quali si rileva un'incidenza del $50 \%$. Incidenze più basse si osservano nei pazienti con linfomi a prognosi favorevole, leucemie, carcinomi della mammella e sarcomi dei tessuti molli [2].

Di recente la European Society for Clinical Nutrition and Metabolism (ESPEN) ha pubblicato una linea guida, "Nutrizione clinica nel cancro", strutturata secondo un diagramma di flusso che copre tutti gli aspetti nutrizionali del cancro trattando i concetti generali di trattamento e gli interventi relativi alle specifiche categorie di trattamento (Fig. 1) [1].

\section{Concetti generali di trattamento}

Dal punto di vista nutrizionale, gli aspetti da considerare nei pazienti affetti da neoplasia maligna sono:

- la verifica, prima del rischio e poi dello stato nutrizionale; 
Fig. 1 Diagramma di flusso relativo ai concetti generali di trattamento e agli interventi nel paziente oncologico

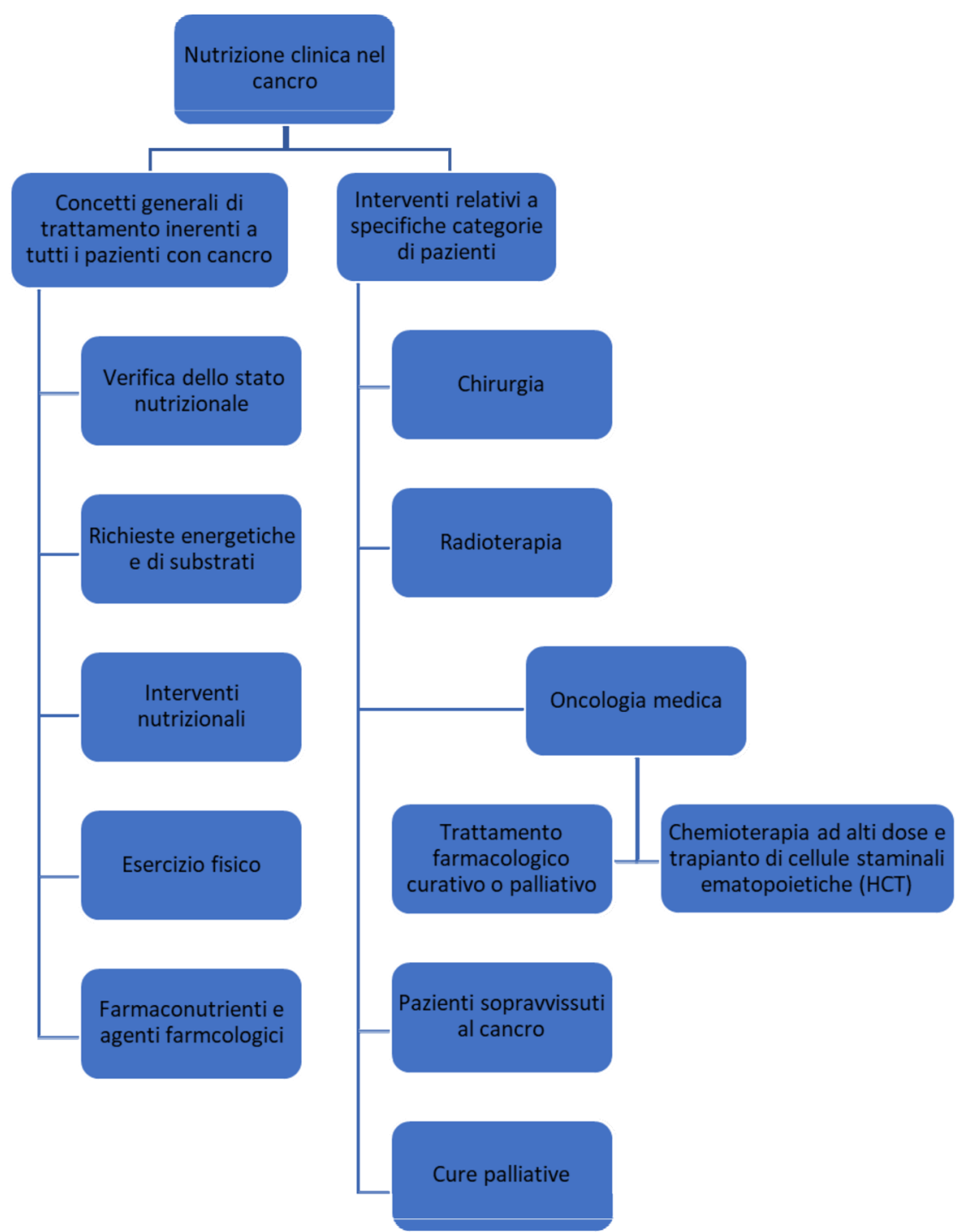

- la prescrizione dell'intervento nutrizionale;

- il monitoraggio dell'apporto nutrizionale e dello stato di nutrizione con una frequenza che è funzione della stabilità della situazione clinico-nutrizionale [1].

\section{Screening nutrizionale}

Il Nutrition Risk Screening 2002 (NRS-2002), il Malnutrition Universal Screening Tool (MUST), il Malnutrition Screening Tool (MST) e il Mini Nutritional Assessment Short Form Revised sono gli strumenti che consentono un'adeguata valutazione del rischio nutrizionale [3] (Figg. 2 e 3).

Lo screening nutrizionale dovrebbe essere effettuato entro le 24-48 ore dall'entrata dei pazienti in reparto e a intervalli regolari (ad esempio ogni settimana) al fine di identificare precocemente i pazienti per i quali dovrebbe essere richiesta una valutazione nutrizionale specialistica [3].

\begin{tabular}{l}
\hline Il BMI è $<20 \mathrm{~kg} / \mathrm{m}^{2}$ ? \\
Il paziente ha perso peso negli ultimi 3 mesi? \\
Il paziente ha ridotto gli introiti alimentari \\
nell'ultima settimana? \\
$\begin{array}{l}\text { Il paziente presenta una patologia acuta grave? } \\
\text { (es. è ricoverato in terapia intensiva) }\end{array}$ \\
\hline
\end{tabular}

Fig. 2 Nutritional Risk Score (NRS)

Riportiamo, ad esempio, le 4 domande dello screening iniziale del NRS-2002, il test più frequentemente usato in ambito ospedaliero. Se solo uno di questi item ha esito positivo si procede con la valutazione dello stato di nutrizione. Se la risposta è no a tutte le domande, il rischio nutrizionale deve essere rivalutato ogni settimana [4].

Il MUST permette, inoltre, di stratificare il rischio nutrizionale a seconda della gravità, indicando anche il percorso successivo. 
Fig. 3 Malnutrition Universal Screening Tool (MUST)

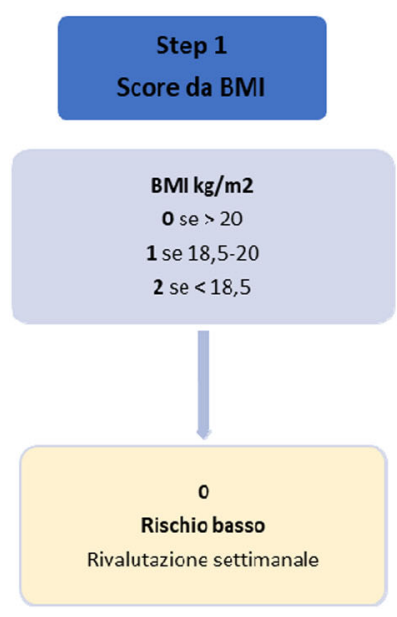

\section{Valutazione dello stato di nutrizione}

La valutazione dello stato nutrizionale dovrebbe essere effettuata nei pazienti risultati a rischio di malnutrizione dal test di screening di cui sopra tramite:

- la rilevazione delle misure antropometriche come il peso corporeo, il calcolo del BMI, l'indice altezza-creatinina e la valutazione della composizione corporea mediante bioimpedenzometria (BIA), Dual X-ray absorptiometry (DXA), Magnetic Resonance Tomography (MRT) o Computed Tomography $(\mathrm{CT})$ a seconda delle disponibilità [4];

- le analisi biochimiche come l'emocromo completo, il profilo lipidico, il compenso glicemico, gli elettroliti, i parametri epatici e renali, l'assetto protidemico (albuminemia, transferrinemia, retinol binding protein, $\mathrm{RBP}$ ), e la proteina $\mathrm{C}$ reattiva, che possono fornire preziose informazioni sullo stato nutrizionale, sulla gravità e sull'attività della malattia. Il dosaggio di vitamine (C, D, E, K, tiamina, B6, B12 e acido folico) e oligoelementi (zinco, selenio e ferro) può aiutare a rilevare carenze nei soggetti cronicamente malnutriti e a monitorare le attuali terapie sostitutive [4];

- la valutazione clinica, che si avvale di una corretta raccolta anamnestica clinica e alimentare del paziente, dell'esame obiettivo e della valutazione dei parametri funzionali (sit-to-stand, 6MWT, hand-grip strength) che sono buoni predittori di esito chirurgico, aumento della durata della degenza ospedaliera e di mortalità a breve e lungo termine [4].

\section{Prescrizione dell'intervento nutrizionale}

Il paziente oncologico ha una spesa energetica a riposo (REE) tendenzialmente aumentata rispetto ai controlli sani confrontabili per età, composizione corporea, attività fisica e calo ponderale [1].
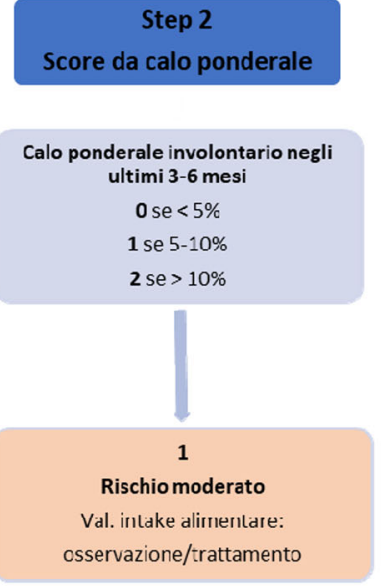
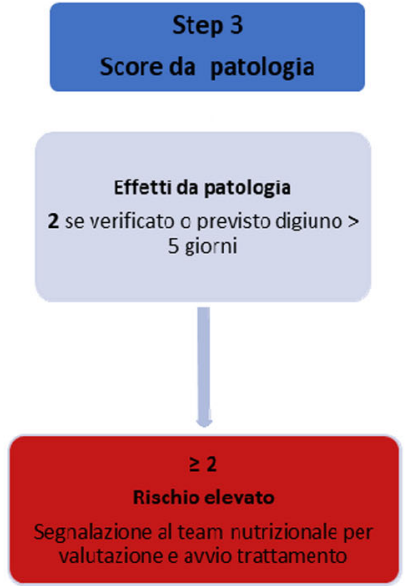

L'intervento nutrizionale corretto necessita di un' adeguata stima di questa spesa energetica per arrivare alla stima della spesa energetica totale (TEE), intesa come la somma della spesa energetica a riposo, dell'attività fisica, dello stress legato alla malattia e della termogenesi indotta dalla dieta e garantirne, quindi, il pieno raggiungimento.

La calorimetria indiretta rimane il gold standard per il calcolo del REE e poi del TEE ai fini di avviare un corretto piano nutrizionale in termini quantitativi sia calorici sia proteici ma, quando non disponibile, è consenso comune stimare le richieste energetiche del malato oncologico tra le 25 e le $30 \mathrm{Kcal} / \mathrm{kg}$ di peso attuale/die con un apporto proteico maggiore di $1 \mathrm{~g} / \mathrm{kg}$ di peso attuale/die potendo raggiungere i $1,5 \mathrm{~g} / \mathrm{kg} / \mathrm{die}[1-5]$.

In merito all'assunzione di vitamine e minerali, viene consigliato di garantire l'assunzione dei Livelli di Assunzione di Riferimento di Energia e Nutrienti (LARN), mentre ne viene scoraggiata l'assunzione ad alte dosi. Si ricordi che le vitamine idrosolubili sono le prime a venire meno con un'alimentazione insufficiente e, a maggior ragione, nel paziente che fa solo utilizzo della nutrizione parenterale [1].

Non esistono, ad oggi, evidenze scientifiche di formulazioni dietetiche come la dieta chetogenica o il digiuno che curino le patologie neoplastiche o ne prevengano le recidive [1].

Qualora l'apporto energetico sia stato meno del 50\% del fabbisogno energetico richiesto per almeno una settimana o il paziente abbia soddisfatto solo il 50-75\% del fabbisogno energetico richiesto per più di 2 settimane, l'uso della nutrizione artificiale (supplementi nutrizionali orali, nutrizione enterale e parenterale) trova indicazione [1].

Il medico che dispone per l'avvio della nutrizione artificiale dovrà essere consapevole dei possibili effetti positivi sul recupero del peso corporeo e sul miglioramento della qualità di vita, che però spesso non si riverberano sulla sopravvivenza del paziente [6,7].

Nei casi di apporti calorici molto scarsi, si raccomanda che l'induzione della rialimentazione avvenga con lenta pro- 
gressione per non incorrere nella sindrome da refeeding, caratterizzata da ipofosfatemia, iposodiemia, ipopotassiemia, ipomagnesiemia, ipoglicemia e ipoproteinemia. Prima e durante il trattamento nutrizionale è indicato supplementare la vitamina B1 alle dosi giornaliere di 200-300 mg; questo perché, se da un lato l'insufficiente apporto alimentare contribuisce a una possibile carenza, questa si aggrava con l'avvio della nutrizione artificiale, dato il suo aumentato consumo nello svolgersi dei diversi processi biologici, principalmente nel metabolismo del glucosio [8].

Infine, non si deve dimenticare l'importanza dell'attività fisica, che si è dimostrata essere sicura e ben tollerata anche per pazienti in stadi avanzati di malattia [1]. Con codesta si intende un' attività domiciliare e supervisionata a moderata intensità (circa 50-75\% della frequenza cardiaca a riposo) per 3 sessioni alla settimana della durata di circa 10-60 minuti, alla quale è consigliato aggiungere un allenamento contro resistenza che sembra più efficace nel mantenere la forza e la massa muscolare [1].

\section{Farmaconutrienti e agenti farmacologici}

Il calo dell'appetito rappresenta una sfida nel paziente oncologico: gli unici farmaci con sufficiente relativa evidenza clinica di efficacia nei pazienti con malattia di grado avanzato sono i corticosteroidi e i progestinici [1].

I primi sono da utilizzare per un arco di tempo limitato (circa 1-3 settimane), in quanto l'effetto oressigeno si esplica in questo lasso di tempo e scompare in poche settimane, mentre sopravvengono gli effetti dannosi a carico del sistema immunitario e della muscolatura a cui si aggiunge, nel lungo termine, l'osteopenia. I secondi portano a un aumento del peso corporeo, ma non della lean body mass (LBM) e sono da utilizzare contestualmente alla valutazione del rischio tromboembolico. L'uso dei corticosteroidi per periodi più prolungati trova un razionale nel trattamento del dolore e della nausea nei pazienti con una aspettativa di vita breve [1].

Dati relativi all'uso dei cannabinoidi non hanno portato a evidenze scientifiche tali da giustificarne l'utilizzo a fini oressigeni, eccetto piccole evidenze derivate da un RCT pilota [1].

Nei pazienti candidati a chemioterapia e a rischio nutrizionale o già malnutriti, la supplementazione con acidi grassi omega 3 viene consigliata per aumentare l'appetito, la LBM e il peso corporeo [5].

In coloro che lamentano un senso di sazietà precoce, è suggerito considerare l'uso di agenti procinetici come la metoclopramide che, a dosaggi di $40-80 \mathrm{mg}$ al die, apporta benefici sulla nausea, ma nessun effetto oressizzante, potendo invece comportare effetti collaterali sul sistema nervoso centrale [1].
Non ci sono ad oggi evidenze circa l'uso di aminoacidi (ad esempio a catena ramificata, la glutamina o l'arginina) o di loro metaboliti (ad esempio il $\beta$-idrossi- $\beta$-metil butirrato, HMB, metabolita della leucina) per l'aumento della massa magra. Nessuna evidenza neanche per l'utilizzo dei FANS e per gli steroidi androgenici.

\section{Interventi relativi a specifiche categorie di pazienti}

Ogni paziente oncologico ha un percorso terapeutico differente e per ogni tipologia di trattamento esistono evidenze su quale sia l'approccio nutrizionale più indicato.

\section{Pazienti candidati all'intervento chirurgico}

Per tutti i pazienti oncologici candidati alla chirurgia curativa o palliativa è raccomandata la gestione entro un programma di recupero detto enhanced recovery after surgery (ERAS). Questo protocollo prevede, tra gli altri, lo screening per la malnutrizione e, qualora il paziente sia ritenuto a rischio, un adeguato programma nutrizionale. (Raccomandazione $\mathrm{C} 1-1$; forza della raccomandazione forte, livello di evidenza e consenso alti) [1].

Le basi nutrizionali del programma comprendono il carico preoperatorio di carboidrati, sotto forma di bevanda da assumere la sera prima e due ore prima dell'intervento (invece che il digiuno) e la ripresa della dieta orale dal primo giorno postoperatorio. Dati di letteratura suggeriscono che l'ERAS consenta una risposta metabolica alla chirurgia ridotta al minimo.

Nei pazienti chirurgici a rischio nutrizionale è raccomandato, oltre a quanto indicato precedentemente, un supporto nutrizionale preoperatorio con integratori nutrizionali liquidi arricchiti con nutrienti specifici (arginina, acidi grassi n-3, nucleotidi) detta, appunto, immunonutrizione [1].

\section{Pazienti che effettuano radioterapia}

Ai pazienti sottoposti a radioterapia, specialmente nei distretti testa-collo, torace e tratto GI, deve essere assicurato un adeguato supporto nutrizionale anche attraverso l'utilizzo di supplementi nutrizionali orali (ONS) al fine di evitare il peggioramento dello stato nutrizionale, scongiurare interruzioni del trattamento e migliorare la qualità di vita (Raccomandazione $\mathrm{C} 2-1$; forza della raccomandazione forte, livello di evidenza moderato, consenso forte) [1].

Questo supporto nutrizionale dovrebbe, in prima istanza, essere garantito tramite l'alimentazione orale eccetto quando, a causa della presenza di mucositi gravi o ostacoli lungo la prima porzione dell'apparato digerente, viene raccomandata la nutrizione enterale tramite l'utilizzo di un sondino naso-gastrico o di una gastrostomia endoscopica percutanea (PEG) [1]. 
Non ci sono, ad oggi, dati consistenti sull'utilizzo della glutamina per prevenire la diarrea, la stomatite, l'esofagite $o$ la tossicità cutanea indotta dalla radioterapia. Nessuna evidenza sull'uso preventivo dei probiotici sull'insorgenza di diarrea.

\section{Pazienti che effettuano chemioterapia curativa o palliativa}

Ci sono dati in letteratura a sostegno della possibilità che la nutrizione enterale possa essere associata a un minor tasso di neutropenia rispetto alla nutrizione parenterale. Anche per questa tipologia di pazienti rimane valido il consiglio di mantenere un opportuno livello di attività fisica e di garantire un adeguato apporto nutrizionale ricorrendo se necessario alla nutrizione artificiale (supplementazione nutrizionale orale, nutrizione enterale o parenterale) [1].

\section{Pazienti che si sottopongono a chemioterapia intensiva e trapianto di cellule staminali ematopoietiche}

Durante la chemioterapia intensiva e dopo il trapianto di cellule staminali, la nutrizione enterale è preferibilmente indicata rispetto alla nutrizione parenterale, in quanto si è dimostrata essere associata a meno complicanze post-trapianto, eccetto in caso di mucositi severe, vomito intrattabile, ileo, malassorbimento severo, diarrea protratta o graft versus host disease sintomatica [1].

Non ci sono, ad oggi, dati in letteratura sufficienti per raccomandare una dieta a bassa carica microbica per più di 30 giorni dopo trapianto allogenico.

\section{Pazienti sopravvissuti al cancro}

Per questa categoria di pazienti è sempre consigliato mantenere un peso normale (BMI 18,5-24,9 kg/m²), il mantenimento di uno stile di vita sano che include l'attività fisica e una dieta a base di verdura, frutta, cereali integrali e a basso contenuto di grassi saturi, carne rossa e alcol [1].

\section{Pazienti con cancro avanzato che non ricevono trattamenti antitumorali (cure palliative)}

Anche i pazienti con cancro avanzato che non ricevono più i trattamenti antitumorali debbono essere valutati dal punto di vista del rischio nutrizionale e dell'apporto di energia e nutrienti.

In questi pazienti è indicato un trattamento nutrizionale solo dopo aver valutato insieme al paziente la prognosi della malattia e l'aspettativa di vita.

In particolare, ci si deve assicurare che la condizione generale del paziente non sia dovuta a un insufficiente apporto nutrizionale, ma agli effetti della malattia rapidamente progressiva. Soprattutto in questi pazienti la valutazione della qualità di vita e l'impatto che l'intervento nutrizionale ha su questa debbono essere attentamente valutati [1].

Informazioni Supplementari La versione online contiene materiale supplementare disponibile su https://doi.org/10.1007/s40619-02201041-4.

\section{Dichiarazioni etiche}

Conflitto di interesse Gli autori Ludovica Cogorno, Eleonora Poggiogalle e Lorenzo M. Donini dichiarano di non avere conflitti di interesse.

Consenso informato Lo studio presentato in questo articolo non ha richiesto sperimentazione umana.

Studi sugli animali Gli autori di questo articolo non hanno eseguito studi sugli animali.

Open Access This article is licensed under a Creative Commons Attribution 4.0 International License, which permits use, sharing, adaptation, distribution and reproduction in any medium or format, as long as you give appropriate credit to the original author(s) and the source, provide a link to the Creative Commons licence, and indicate if changes were made. The images or other third party material in this article are included in the article's Creative Commons licence, unless indicated otherwise in a credit line to the material. If material is not included in the article's Creative Commons licence and your intended use is not permitted by statutory regulation or exceeds the permitted use, you will need to obtain permission directly from the copyright holder. To view a copy of this licence, visit http://creativecommons.org/licenses/by/4.0/.

\section{Bibliografia}

1. Muscaritoli M, Arends J, Bachmann P et al (2021) ESPEN practical guideline: clinical nutrition in cancer. Clin Nutr 40(5):2898-2913

2. Huhmann MB, August DA (2008) Review of American Society for Parenteral and Enteral Nutrition (ASPEN) clinical guidelines for nutrition support in cancer patients: nutrition screening and assessment. Nutr Clin Pract 23(2):182-188

3. Arends J, Bachmann P, Baracos V et al (2017) ESPEN guidelines on nutrition in cancer patients. Clin Nutr 36(1):11-48

4. Reber E, Gomes F, Vasiloglou MF et al (2019) Nutritional risk screening and assessment. J Clin Med 8(7):1065

5. Prado CM, Laviano A, Gillis C (2021) Examining guidelines and new evidence in oncology nutrition: a position paper on gaps and opportunities in multimodal approaches to improve patient care. Support Care Cancer. https://doi.org/10.1007/s00520-021-06661-4

6. Baldwin C, Spiro A, Ahern R, Emery PW (2012) Oral nutritional interventions in malnourished patients with cancer: a systematic review and meta-analysis. J Natl Cancer Inst 104(5):371-385

7. Laviano A, Di Lazzaro L, Koverech A (2018) Nutrition support and clinical outcome in advanced cancer patients. Proc Nutr Soc 77(4):388-393

8. Polegato BF, Pereira AG, Azevedo PS et al (2019) Role of thiamin in health and disease. Nutr Clin Pract 34(4):558-564

Nota della casa editrice Springer Nature rimane neutrale in riguardo alle rivendicazioni giurisdizionali nelle mappe pubblicate e nelle affiliazioni istituzionali. 